M. GLASNER

KODAI MATH. J.

10 (1987), 307-313

\title{
DIRICHLET PRINCIPLE FOR THE ROYDEN COMPACTIFICATION
}

\author{
By Moses GLASNer
}

Consider a hyperbolic Riemann surface $R$. Given some notion of vanishing of functions on a portion of the ideal boundary of $R$ and a suitable function $f$ on $R$ with finite Dirichlet integral, $D(f)=\int_{R} d f \wedge * d f<\infty$, consider the family of Dirichlet finite functions $\widetilde{\mho}_{f}$ consisting of functions $\phi$ such that $\phi-f$ "vanishes" at the ideal boundary. A Dirichlet principle is the statement that there exists a unique harmonic function $h_{f}$ in the family $\mathfrak{F}_{f}$ such that

$$
D\left(h_{f}\right)=\inf \left\{D(\phi) ; \phi \in \widetilde{F}_{f}\right\} .
$$

Several notions of vanishing have been considered. Royden [6] and Kuramochi [4] introduced compactifications of $R$ and required functions to have the value 0 on certain subsets of the additions to $R$. Brelot [1] required functions to have 0 limit along almost every line in a bundle of Green's lines whereas Ohtsuka [5] required the limit to be 0 along almost every curve in a family of curves of finite extremal length tending to the ideal boundary.

The latter three works allow free boundary values; that is, in the definition of $\mathfrak{r}_{f}$ vanishing is required only at a portion of the potential-theoretically significant part of the ideal boundary. In this case the minimizing function may not agree with the given function on the prescribed portion and thus the notion of vanishing must allow for exceptions. Although capacities have been studied on the Royden boundary (cf. [8]), essentially only harmonic measure has been used extensively for specifying exceptions there. But the latter is not sufficiently sensitive to the Dirichlet integral to be used in formulating a Dirichlet principle. In this paper we show that the definition of Dirichlet capacity introduced in [3] leads to the Dirichlet principle with free boundary values on the Royden compactification.

1. We use the standard notations and results associated with the Royden boundary theory as presented in [7]. The Royden compactification of $R$ is denoted by $R^{*}$. We will use the symbols $\operatorname{cl}(A)$ and $\partial A$ to denote closure and

The author is grateful to the Institute of Mathematics at the Hebrew University of Jerusalem for its hospitality during his sabbatical.

Received March 6, 1987. 
boundary in $R$, while reserving the symbol $\bar{A}$ for the closure of $A$ in $R^{*}$.

We begin by recalling the definition of Dirichlet capacity given in [3]. Since we will be dealing with this capacity exclusively we will simply call it capacity here. Fix a regular boundary neighborhood $W$ of $R$, i.e., $R-W$ is compact and $\partial W$ is piecewise analytic. We denote by $M(W ; \partial W)$ the functions in the Royden algebra $M(R)$ which vanish on $R-W$. For an open set $O \subset R$ with $\mathrm{cl}(\theta) \subset W$ consider the family

and define

$$
\mathcal{E}=\{\phi \in M(W ; \partial W) ; \phi \mid 0 \geqq 1\}
$$

$$
d(\mathcal{O})=\inf \{D(\phi) ; \phi \in \mathcal{E}\}
$$

It is easy to see that $d$ is an increasing real-valued function on the open subsets of $W$ with closures in $W$. Set $W^{*}=\bar{W}-\partial W$. For a compact set $K$ in $W^{*}$ we set

$$
c(K)=\inf \left\{d(U \cap R) ; U \text { open in } W^{*}, K \subset U, \operatorname{cl}(U \cap R) \subset W\right\} .
$$

It is easy to see that $c(\cdot)$ is a capacity in the sense of Choquet (cf. [3]). For an open $\theta \subset R^{*}, c(\theta)$ is defined by

$$
c(\mathcal{O})=\sup \left\{c(K) ; K \text { is compact in } W^{*}, K \subset \mathcal{O}\right\} .
$$

For an arbitrary subset $A$ of $W^{*}$, with $\bar{A} \subset W^{*}$, the inner and outer capacities of $A$ are defined in the usual manner and it is called capacitable if the two agree. By [3, Proposition 11] compact sets in $W^{*}$ are capacitable and therefore so is any countable union of compact sets of capacity 0.

2. If $K$ is a compact subset of the Royden harmonic boundary $\Delta$, then it is convenient to have the somewhat simpler expression for $c(K)$ given in the following

LEMMA. There is a unique function $u_{K} \in H B D(W ; \partial W)$ which is the BD-limit of any sequence minimizing the Dirichlet integral in the family

$$
\mathcal{C}=\{\phi \in M(W ; \partial W) ; \phi \mid K \geqq 1\} .
$$

Moreover, $c(K)=D\left(u_{K}\right)$.

We shall call the function $u_{K}$ the capacitary potential for $K$. Also note that $u_{K}$ may be less than 1 at some points of $K$. (From the proof of Proposition 4 it will follow that these points are at most a set of capacity 0.) For the proof set $d=\inf \{D(\phi) ; \phi \in \mathcal{C}\}$. Let $\left\{u_{n}\right\} \subset \mathcal{C}$ be a minimizing sequence, i.e., $d=$ $\lim D\left(u_{n}\right)$. By replacing $u_{n}$ by $u_{n} \cap 1$, we may assume that $0 \leqq u_{n} \leqq 1$; in particular, $u_{n} \mid K=1$. Then by replacing $u_{n}$ by its harmonic projection on $W$ (cf. [7]), we may also assume that $u_{n} \in H B D(W ; \partial W)$. Since the family $\mathcal{C}$ is convex, any minimizing sequence in $\mathcal{C}$ is $D$-Cauchy. We conclude that there is a func- 
tion $u_{K} \in H B D(W ; \partial W)$ such that $u_{K}=B D-\lim u_{n}$ and $d=D\left(u_{K}\right)$.

To complete the proof we must show that $d=c(K)$. Set $a_{n}=(n-1) / n$ and $U_{n}=\left\{p \in R^{*} ; u_{n}(p)>a_{n}\right\}$, an open subset of $R^{*}$ containing $K$. Also set $f_{n}=$ $\left(u_{n} \cap a_{n}\right) / a_{n}$. Then by (2) and (3), $D\left(f_{n}\right) \geqq d\left(U_{n} \cap R\right) \geqq c(K)$. Consequently, $d=$ $\lim D\left(u_{n}\right)=\lim D\left(f_{n}\right) \geqq c(K)$.

For the reverse inequality, by (3) we can choose a sequence of open sets $\left\{\mathcal{O}_{n}\right\}$ in $R^{*}$ with $\operatorname{cl}\left(\mathcal{O}_{n} \cap R\right) \subset W, K \subset \mathcal{O}_{n}$ and $c(K)=\lim d\left(\mathcal{O}_{n} \cap R\right)$. Then by (2) we choose $\left\{\phi_{n}\right\} \subset M(W ; \partial W)$ with $\phi_{n} \mid \Theta_{n} \cap R \geqq 1$ and $D\left(\phi_{n}\right) \leqq d\left(\Theta_{n} \cap R\right)+n^{-1}$. By the denseness of $\mathcal{O}_{n} \cap R$ in $\mathcal{O}_{n}$, we also have $\phi_{n} \mid \Theta_{n} \geqq 1$ and hence $\phi_{n} \in \mathcal{C}$. Thus $d \leqq D\left(\phi_{n}\right) \leqq d\left(\mathcal{O}_{n} \cap R\right)+n^{-1}$, which gives $d \leqq c(K)$.

\section{We need another technical.}

LEMMA. If $\phi \in M(W ; \partial W)$ with $\psi \mid K \geqq 0$, then

$$
D\left(u_{K}, \phi\right) \geqq 0 .
$$

For the proof take a minimizing sequence $\left\{u_{n}\right\} \subset \mathcal{C}$. Then for every $\varepsilon>0$ and every positive integer $n$ the function $u_{n}+\varepsilon \phi \in \mathcal{C}$. Thus, $D\left(u_{K}\right) \leqq D\left(u_{n}+\varepsilon \psi\right)$ $=D\left(u_{n}\right)+2 \varepsilon D\left(u_{n}, \psi\right)+\varepsilon^{2} D(\psi)$. By letting $n \rightarrow \infty$ we see that $0 \leqq 2 D\left(u_{n}, \psi\right)+\varepsilon D(\psi)$, and then letting $\varepsilon \rightarrow 0$ establishes (4).

4. The following characterization of the compact subsets of $\Delta$ with positive capacity is the key to relating convergence of boundary values to convergence on $R$.

Proposition. Let $K$ be a compact subset of $\Delta$. Then $c(K)>0$ if and only if every sequence $\left\{g_{n}\right\} \subset M(W ; \partial W)$, with $\left\{D\left(g_{n}\right)\right\}$ bounded, $0 \leqq g_{n} \leqq 1, g_{n} \mid K=1$ and $g_{0}=B-\lim g_{n}$ existing, satisfies

$$
\max _{K} g_{0}=1
$$

For the sufficiency note that the minimizing sequence $\left\{u_{n}\right\}$ used in the proof of Lemma 2 has all the stated properties of the sequence $\left\{g_{n}\right\}$ with $g_{0}=u_{K}$. Thus (5) implies that $u_{K} \not \equiv 0$ and thus $c\left(u_{K}\right)=D\left(u_{K}\right)>0$.

For the proof of the necessity let $\left\{g_{n}\right\}$ be a sequence having the properties given in the proposition except that it does not satisfy (5); i. e., $\alpha=\max _{K} g_{0} \in$ $[0,1)$. Note that by Kawamura's lemma (cf. [7]), $g_{0} \in M(W ; \partial W)$ and $\left\{g_{n}\right\}$ converges to $g_{0} D$-weakly. Let $\left\{u_{n}\right\} \subset \mathcal{C}$ be a minimizing sequence chosen with $u_{n} \mid K=1$ as in the proof of Lemma 2. Since $g_{n}-u_{n} \mid K=0$, Lemma 3 implies that $D\left(u_{K}, g_{n}-u_{n}\right)=0$ and by letting $n \rightarrow \infty$ we have

$$
D\left(u_{K}, g_{0}-u_{K}\right)=0 .
$$

On the other hand, since $\alpha g_{n}-g_{0} \mid K \geqq 0$, Lemma 3 implies that $D\left(u_{K}, \alpha g_{n}-g_{0}\right) \geqq 0$ 
and by letting $n \rightarrow \infty$ we obtain $(\alpha-1) D\left(u_{K}, g_{0}\right) \geqq 0$, or,

$$
D\left(u_{K}, g_{0}\right) \leqq 0 .
$$

Subtracting (6) from (7) gives $D\left(u_{K}\right) \leqq 0$, which means that $c(K)=0$.

5. We are ready to establish a preliminary version of the Dirichlet principle on the Royden compactification.

Proposition. Given a compact set $K \subset \Delta$ with $c(K)>0$ and $f \in M(R)$, consider the family

$$
\mathfrak{F}_{f}=\{\phi \in M(R) ; \phi=f \text { q.e. on } K\} \text {. }
$$

There exists a unique harmonic function $h_{f} \in \mathfrak{F}_{f}$ satisfying (1).

Here and throughout, we say that a property holds q.e. on a set if it holds on the set except for a subset of capacity 0 .

The uniqueness of $h_{f}$ follows from (1). Indeed, as in the proof of Lemma 3 it is easy to see that if a function $h^{\prime} \in \widetilde{F}_{f}$ satisfies (1), then $D\left(h^{\prime}, \phi\right)=0$ for every $\phi \in M(R)$ with $\phi=0$ q. e. on $K$. Thus $D\left(h^{\prime}, h^{\prime}-h_{f}\right)=0$, and by symmetry, $D\left(h_{f}, h^{\prime}-h_{f}\right)=0$. This implies that $h^{\prime}-h_{f}$ is a constant, which must be 0 since $h^{\prime}-h_{f}=0$ on a nonempty subset of $K$.

For the proof of the existence choose a minimizing sequence $\left\{h_{n}\right\} \subset \mathfrak{F}_{f}$. We may assume that $h_{n}$ is harmonic on $R$ and that $\min _{K} f \leqq h_{n} \leqq \min _{K} f$. Thus by the Harnack principle a subsequence, again denoted by $\left\{h_{n}\right\}$, has $h_{f}=B-\lim h_{n}$. Since $\mathfrak{F}_{f}$ is convex, $\left\{h_{n}\right\}$ is $D$-Cauchy and hence we even have $h_{f}=B D-\lim h_{n}$. In particular, $h_{f}$ satisfies (1). It remains to show that $h_{f} \in \widetilde{\mho}_{f}$.

For each $n$, we may choose $K_{n} \subset K$ such that $h_{n}\left|K_{n}=f\right| K_{n}$ and $c\left(K_{n}\right)=0$. Set $K_{\infty}=\cup K_{n}$ and $F=K-K_{\infty}$. Then $c\left(K_{\infty}\right)=0$ and

$$
h_{n}|F=f| F, \quad \text { for every } n \text {. }
$$

We complete the proof by showing that if $E$ is a nonempty compact subset of $\left\{p \in F ; h_{f}(p) \neq f(p)\right\}$, which is an $F_{\sigma}$-set in $R^{*}$, then $c(E)=0$. Since any such $E$ is the union of a compact set in $\left\{p \in F ; h_{f}(p)>f(p)\right\}$ with one in $\{p \in F$; $\left.h_{f}(p)<f(p)\right\}$, we may assume that it is contained in the latter, as the other situation is entirely analogous. Set $m=\min _{E}\left(f-h_{f}\right)$. Clearly, $m>0$. Also define $v_{n}=1-\left(f-h_{n}\right)$ and $v_{0}=1-\left(f-h_{f}\right)$. Then $\left\{D\left(v_{n}\right)\right\}$ is bounded, $v_{0}=B-\lim v_{n}$, $v_{n} \mid E=1$ and $\max _{E} v_{0}=1-m<1$. By replacing $v_{n}$ by $\left(v_{n} \cap 1\right) \cup 0$, and $v_{0}$ by $\left(v_{0} \cap 1\right)$ $\cup 0$, we can ensure that $0 \leqq v_{n} \leqq 1$ without disturbing the above properties.

Let $w \in M(W ; \partial W)$ such that $0 \leqq w \leqq 1$ and $w \mid \Delta=1$ and set $g_{n}=w v_{n}, g_{0}=w v_{0}$. We see that $g_{n}, g_{0} \in M(W ; \partial W),\left\{D\left(g_{n}\right)\right\}$ is bounded, $0 \leqq g_{n} \leqq 1, g_{0}=B-\lim g_{n}$, $g_{n} \mid E=1$ and $\max _{E} g_{0}=(1-m) \cup 0<1$. Thus by Proposition 4 , we arrive at $c(E)=0$. 
6. The mapping of $f$ to the minimizing function given by Proposition 5 is linear. In addition, it is order preserving according to the following

Lemma. Consider a compact $K \subset \Delta$ with $c(K)>0$ and $f, g \in M(R)$. If $f \geqq g$ q.e. on $K$, then $h_{f} \geqq h_{g}$.

For the proof assume that the open set $\mathcal{O}^{*}=\left\{p \in R^{*} ; h_{g}(p)>h_{f}(p)\right\}$ is not empty. Then $\mathcal{O}^{*} \cap K$ has capacity 0 . Consider the function

$$
h^{\prime}=\left\{\begin{array}{lll}
h_{g} & \text { on } & \mathcal{O}^{*} \cap R, \\
h_{f} & \text { on } & R-\mathcal{O}^{*} .
\end{array}\right.
$$

Since $h^{\prime}=f$ q.e. on $K, h^{\prime} \in \mathfrak{F}_{f}$. Thus, $D\left(h^{\prime}\right)>D\left(h_{f}\right)$, which implies that

$$
D_{\mathcal{O} * \cap R}\left(h_{g}\right)>D_{\mathcal{O} * \cap R}\left(h_{f}\right) \text {. }
$$

The function

$$
h^{\prime \prime}=\left\{\begin{array}{lll}
h_{f} & \text { on } & \mathcal{O}^{*} \cap R, \\
h_{g} & \text { on } & R-\mathcal{O}^{*} .
\end{array}\right.
$$

belongs to $\mathfrak{F}_{g}$ and in view of (9) we must have $D\left(h_{g}\right)>D\left(h^{\prime \prime}\right)$. This contradiction establishes the assertion.

7. Our goal is to extend the Dirichlet principle given in Proposition 5 to the case of an arbitrary Dirichlet function $f$ (cf. [2]) on $R$. For this purpose we first consider the extended Royden class $\tilde{M}(R)$ (cf. [7]), the set of continuous Dirichlet functions on $R$, and establish the Dirichlet principle for a nonnegative $f \in \tilde{M}(R)$.

First note that for $f \in \tilde{M}(R)$, the set $S=\left\{p \in R^{*} ;|f|(p)=\infty\right\}$ has capacity 0 . To see this simply note that $S$ is a compact subset of $\Delta$ and apply Proposition 4 with $g_{n}=(|f| \cap 1) / n$ and $g_{0}=0$.

LEMMA. Given a compact set $K \subset \Delta$ with $c(K)>0$ and a nonnegatıve $f \in \tilde{M}(R)$, consider the family

$$
\widetilde{F}_{f}=\{\phi \in \tilde{M}(R) ; \phi=f \text { q.e. on } K\} \text {. }
$$

There exists a unique harmonic function $h_{f} \in \mathfrak{F}_{f}$ satisfying (1).

The proof of the uniqueness is as in Proposition 5. For the existence let $u_{k} \in \mathfrak{F}_{f \cap k}, \quad(k=1,2, \cdots)$ be the function which minimizes the Dirichlet integral. Then Lemma 6 implies that $0 \leqq u_{k} \leqq u_{k+1}$. By the Harnack principle either $C$ - $\lim u_{k}$ is $\infty$ or a harmonic function $h_{f}$. Assume that $\infty$ is the limit. Fix a compact set $E \subset K$ such that $c(E)>0$ and $u_{k}=f \cap k$ on $E$. Note that $D\left(u_{k}\right) \leqq D(f \cap k) \leqq$ $D(f)$. Define 


$$
v_{k}=\left(\left(1+\left(u_{k}-f \cap k\right)\right) \cap 1\right) \cup 0 .
$$

Then it is easy to see that $0 \leqq v_{k} \leqq 1,\left\{D\left(v_{k}\right)\right\}$ is bounded, $v_{k} \mid E=1$ and $0=B$ $-\lim v_{k}$. Let $w \in M(W ; \partial W)$ such that $0 \leqq w \leqq 1$ and $w \mid \Delta=1$ and set $g_{k}=w v_{k}$, $g_{0}=0$. By Proposition 4 we arrive at the contradiction that $c(E)=0$ and we have established that $h_{f}=C-\lim u_{k} \in H D(R)$.

Since $h_{f} \geqq u_{k}, h_{f}|K \geqq f \cap k| K$ and consequently, $h_{f}|K \geqq f| K$. We claim that in fact $h_{f}=f$ q.e. on $K$. To show this we take a nonempty compact set $E \subset\left\{p \in K ; f(p)<h_{f}(p)<\infty\right\}$ and show that $c(E)=0$. For this purpose set $m=\min _{E} h_{f}-f$, and

$$
\begin{aligned}
& v_{k}=\left(\left(1-\left(u_{k}-f \cap k\right)\right) \cap 1\right) \cup 0, \\
& v_{0}=\left(\left(1-\left(h_{f}-f\right)\right) \cap 1\right) \cup 0 .
\end{aligned}
$$

We again have $0 \leqq v_{k} \leqq 1,\left\{D\left(v_{k}\right)\right\}$ bounded, $v_{k} \mid E=1$ and $v_{0}=B-\lim v_{k}$. Set $g_{k}=w v_{k}, \quad g_{0}=w v_{0}$. Since $\max _{E} g_{0}=(1-m) \cup 0<1$, Proposition 4 implies that $c(E)=0$.

Having established that $h_{f} \in \mathfrak{F}_{f}$, we still need to show that $h_{f}$ satisfies (1). Let $\left\{h_{n}\right\} \subset \mathfrak{F}_{f}$ be a minimizing sequence. Then $h_{n} \cap k \in \mathfrak{F}_{f \cap k}$ and consequently $D\left(u_{k}\right) \leqq D\left(h_{n} \cap k\right) \leqq D\left(h_{n}\right)$. By Fatou's lemma we obtain $D\left(h_{f}\right) \leqq D\left(h_{n}\right)$, which on letting $n \rightarrow \infty$ implies (1).

\section{We are ready to establish the main result.}

THEOREM. Given a compact set $K \subset \Delta$ with $c(K)>0$ and a Dirichlet function $f$ an $R$, consider the family

$$
\widetilde{\mho}_{f}=\{\phi ; \phi \text { is a Dirichlet function, } \phi=f \text { q.e. on } K\} \text {. }
$$

There exists a unique harmonic function $h_{f} \in \mathfrak{F}_{f}$ satisfying (1).

If $f$ is not continuous on $R$, then we replace it by its harmonic projection on $R$ without disturbing $\widetilde{F}_{f}$ (cf. [2]), i. e., we may assume that $f \in \tilde{M}(R)$. Moreover, since taking the harmonic projection does not increase the Dirichlet integral, we may also assume that $\mathfrak{\mho}_{f} \subset \tilde{M}(R)$. For each positive integer $n$ consider the minimizing function $u_{n} \in \mathfrak{F}_{(f \cup-n) \cap n}$. By Lemma 7 we may also consider the minimizing function $h_{|f| \cap n} \in \mathscr{F}_{|f| \cap n}$. We have seen in the proof that $h_{|f|}$, the minimizing function in $\mathfrak{F}_{|f|}$, satisfies $h_{|f|} \geqq h_{|f| \cap n}$. Thus by Lemma 6

$$
-h_{|f|} \leqq-h_{|f| \cap n} \leqq u_{n} \leqq h_{|f| \cap n} \leqq h_{|f|} \text {. }
$$

By the Harnack principle $h_{f}=C-\lim u_{n}$ exists and is harmonic. Let $\left\{h_{k}\right\} \subset \widetilde{F}_{f}$ be a minimizing sequence. Then by Fatou's lemma

$$
D\left(h_{f}\right) \leqq \underline{\lim } D\left(u_{n}\right) \leqq \lim D\left(\left(h_{k} \cup-n\right) \cap n\right)=D\left(h_{k}\right),
$$

in particular, $h_{f} \in \tilde{M}(R)$. 
We now show that $h_{f} \in \mathfrak{F}_{f}$. By letting $n \rightarrow \infty$ in (10) we see that $\left|h_{f}\right| \leqq h_{|f|}$. Thus, $\left|h_{f}\right||K \leqq| f|| K$. Since $f$ is finite q. e. on $K$, it suffices to show that any nonempty compact set $E \subset\left\{p \in K ; h_{f}(p)<f(p)<\infty\right\}$ has capacity 0 . Set $m=$ $\min _{E} f-h_{f}$, and

$$
\begin{aligned}
& v_{n}=\left(\left(1-\left(u_{n}-(f \cup-n) \cap n\right)\right) \cap 1\right) \cup 0 . \\
& v_{0}=\left(\left(1-\left(h_{f}-f\right)\right) \cap 1\right) \cup 0 .
\end{aligned}
$$

We see that $0 \leqq v_{n} \leqq 1,\left\{D\left(v_{n}\right)\right\}$ is bounded, $v_{n} \mid E=1$ and $v_{0}=B-\lim v_{n}$. Set $g_{n}=w v_{n}, g_{0}=w v_{0}$. Since $\max _{E} g_{0}=(1-m) \cup 0<1$, Proposition 4 implies that $c(E)=0$.

Finally, letting $k \rightarrow \infty$ in (11) establishes (1) and completes the proof.

\section{REFERENCES}

[1] M. BRELOT, Étude et extensions du principe de Dirichlet, Ann. Inst. Fourier 5 (1955), 371-419.

[2] C. Constantinescu And A. CoRnea, Ideale Ränder Riemannscher Flächen, Springer-Verlag, 1963.

[3] M. Glasner AND M. NAKaI, Singular points on compactifications of a Riemann surface, Israel J. Math. 55 (1986), 15-32.

[4] Z. KURAMOCHI, On the ideal boundaries of abstract Riemann surfaces, Osaka Math. J. 10 (1958), 83-102.

[5] M. OнтsukA, Dirichlet principle on Riemann surfaces, J. Analyse Math. 19 (1967), 295-311.

[6] H. Royden, On the ideal boundary of a Riemann surface. Contributions to the theory of Riemann surfaces, Princeton Univ. Press, 1953, 107-109.

[7] L. SARIO AND M. NAKaI, Classification theory of Riemann surfaces, SpringerVerlag, 1970.

[8] H. TANaKa, On Kuramochi's Function-theoretic Separative Metrics on Riemann Surfaces, J. Sci. Hiroshima Univ. Ser. A-1 32 (1968), 309-330.

Department of Mathematics

PenNsylvania State University

UNIVERSITY PARK, PA 16802

USA 